\title{
Upscaling of land-surface parameters through direct moment propagation
}

\author{
H. Kunstmann \\ Institute for Meteorology and Climate Research (IMK-IFU), Forschungszentrum Karlsruhe, Kreuzeckbahnstraße 19, 82467 \\ Garmisch-Partenkirchen, Germany
}

Received: 7 January 2005 - Revised: 1 August 2005 - Accepted: 1 September 2005 - Published: 16 December 2005

\begin{abstract}
A new methodology is presented that allows the upscaling of land surface parameters of a Soil-VegetationAtmosphere-Transfer (SVAT) Model. Focus is set on the proper representation of latent and sensible heat fluxes on grid scale at underlying subgrid-scale heterogeneity. The objective is to derive effective land surface parameters in the sense that they are able to yield the same heat fluxes on the grid scale as the averaged heat fluxes on the subgrid-scale. A combination of inverse modelling and Second-Order-FirstMoment (SOFM) propagation is applied for the derivation of effective parameters. The derived upscaling laws relate mean and variance (first and second moment) of subgridscale heterogeneity to a corresponding effective parameter at grid-scale. Explicit upscaling relations are exemplary derived for a) roughness length, $b$ ) wilting point soil moisture, and c) minimal stomata resistance. It is demonstrated that the SOFM-Method yields congruent results to corresponding Monte Carlo simulations. Effective parameters were found to be independent of driving meteorology and initial conditions.
\end{abstract}

\section{Introduction}

Mesoscale distributed hydrological models as well as process-based regional climate models often use grid resolutions that are not able to account for detailed land surface heterogeneity (e.g. soil, vegetation and land surface properties). The impact of this subgrid-scale heterogeneity usually is not accounted for. Land surface information, however, often is available in higher spatial resolution than the specific model resolution (e.g. via satellite data) and the coarse model resolution is only due to limited CPU resources. If subgridscale effects shall be accounted for on grid-scale, aggregation techniques have to be applied that allow the derivation of effective model parameters.

Correspondence to: H. Kunstmann

(harald.kunstmann@imk.fzk.de)
The upscaling of land surface parameters in this study relates to the physical description of energy and water balance at the land surface according to the equations of the Soil-Vegetation-Atmosphere-Transfer (SVAT) model of the Oregon State University (OSU-LSM) (Ek and Mahrt, 1991; Chen and Dudhia, 2001). The OSU-LSM provides the lower boundary condition of the non-hydrostatic mesoscale meteorological model MM5 (Grell et al., 1994). MM5 usually is applied in horizontal resolutions of $10 \times 10-50 \times 50 \mathrm{~km}^{2}$. This resolution in most cases is too coarse to account for variability in surface parameters like albedo, emissivity, roughness length or vegetation parameters like stomatal resistances. SVAT-models, both in stand-alone versions and those coupled to regional climate models, provide tabulated values for land surface parameters dependent on soil and vegetation type, however, independent of scale (i.e. horizontal model resolution). The question arises, how these land surface parameters must be chosen dependent on the scale (i.e. the horizontal model resolution) such that modelled heat fluxes at grid scale equal aggregated heat fluxes at subgrid scale.

Explicit scaling laws for central land surface parameters are derived that relate mean (1st moment) and standard deviation (2nd moment) of subgrid scale parameter distribution to a corresponding effective parameter value at grid scale. Effective parameters are derived by a combination of first moment propagation and inverse modelling. It is in particular shown that the Monte Carlo based approach (as introduced by Intsiful, 2004) and the direct first moment propagation approach (based on a Taylor-Series expansion) of this work yields identical results.

\section{The SVAT model - brief overview}

The SVAT model applied in this study closely follows the approach of Ek and Mahrt (1991) and Chen and Dudhia (2001). It solves the energy balance

$R_{n}=G+\lambda E+H$ 
with $R_{n}$ : net radiation $\left[\mathrm{Wm}^{-2}\right], G$ : ground heat flux $\left[\mathrm{Wm}^{-2}\right], \lambda E$ : latent heat flux $\left[\mathrm{Wm}^{-2}\right], H$ : sensible heat flux $\left[\mathrm{Wm}^{-2}\right]$.

Net radiation is obtained by

$R_{n}=(1-\alpha) S W_{i n}+L W_{i n}-\varepsilon \cdot \sigma \cdot t_{\mathrm{sfc}}^{4}$

with $\alpha$ : albedo [.], $\varepsilon$ : emissivity [.], $S W_{\text {in }}$ : incoming shortwave radiation $\left[\mathrm{Wm}^{-2}\right], \mathrm{LW}_{i n}$ : incoming longwave radiation $\left[\mathrm{Wm}^{-2}\right], \sigma$ : Stefan-Boltzmann constant $\left[5.6710^{-8} \mathrm{Wm}^{-2} \mathrm{~K}^{-4}\right], t_{\mathrm{sfc}}$ : temperature of land surface $[\mathrm{K}]$.

The model has the prognostic variables soil temperature, volumetric soil water content, canopy water content and the diagnostic variables sensible heat flux, latent heat flux (from canopy, stomata and bare soil), ground heat flux, infiltration excess. The soil is discretized in four layers with vertical resolutions $10 \mathrm{~cm}, 30 \mathrm{~cm}, 60 \mathrm{~cm}$, and $100 \mathrm{~cm}$. Soil thermodynamics is accounted for by solving the heat flux equation, in which volumetric heat capacity depends on actual volumetric soil content. Soil moisture dynamics in the unsaturated zone is described by solving the diffusive form of Richard's equation. Soil hydraulic conductivity, hydraulic conductivity and matric potential nonlinearly depend on the ClappHornberger parameter $b$ (a curve fitting parameter dependent on soil type). Canopy transpiration is calculated dependent on vegetation fraction, canopy resistance, canopy water content and potential evaporation. Canopy resistance nonlinearly depends on leaf area index (LAI), minimum $\left(R c_{\min }\right)$ and maximum stomatal resistance, solar insolation, vapour pressure, atmospheric temperature and soil moisture content. When the soil moisture exceeds the field capacity $\theta_{\text {ref }}$, transpiration is not regulated by soil moisture deficit. When the soil moisture is less than wilting point soil moisture $\theta_{\text {wilt }}$, soil water deficit prevents transpiration. Aerodynamic roughness length $\left(z_{0 \mathrm{~m}}\right)$ determines the zero-wind level in the vertical logarithmic wind profile of the planetary boundary layer (PBL); as it non-linearly impacts canopy resistance it is very sensitive to latent heat flux.

The SVAT-model is programmed in Mathematica (Wolfram, 2004) and applied and validated using forcing and observation data from Meyers/NOAA measurement site in Champaign, Illinois. The measurement site is located at $88.37^{\circ} \mathrm{W}$ and $40.01^{\circ} \mathrm{N}$. Data are available for the scientific community and can be retrieved via ftp://ftp.ncep.noaa.gov. The site is characterised by soil type "silty loam" and vegetation type "groundcover only". This study focuses on Julian days 195-200 in 1998. A comparison between modelled and observed sensible and latent heat fluxes are given in Figs. 1a, and b. The SVAT model in general reproduces well observed daily variation of heat fluxes. Time resolution $\Delta t$ was $15 \mathrm{~min}$.

\section{Upscaling methodology}

Figure 2 illustrates the general methodology applied for deriving effective parameters. In a square of $n$ gridpoints at subgrid scale, every grid point $i$ is characterised by a different land surface parameter $p_{i}(i=1, \ldots, n)$. In our case, $p$ can for example represent albedo, emissivity, roughness length or any other land surface parameter of interest. The SVAT model calculates the energy and water balance at the land surface and provides modelled values for heat fluxes $F$ for every time step $\Delta t$. In the following it is assumed that the $n$ parameters are normally distributed:

$p=N\left(\mu_{p}, \sigma_{p}\right)$

The mean (i.e. spatially averaged) total heat fluxes $F$ ( $F$ can indicate either sensible heat flux $H$, latent heat flux $\lambda E$ or ground heat flux $G$ ) within a given time interval $\left[t_{0}, t_{\max }\right]$, i.e. the first moment of $F$, is obtained by

$\hat{F}\left(\mu_{p}, \sigma_{p}\right) \approx \frac{1}{n} \sum_{i=1}^{n} F_{i}\left(p_{i}\right)$

where

$F_{i}=\sum_{t=t_{0}}^{t=t_{\max }} F_{i}(t)$

indicate the temporal aggregated heat flux at every grid point $i$ over total time interval $\left[t_{0}, t_{\max }\right]$.

Alternatively to the Monte Carlo approach of (3) and the aggregation according to (4), direct propagation of the first moment of $F$ can be performed. This is achieved by

$$
\begin{aligned}
\hat{F}\left(\mu_{p}, \sigma_{p}\right)=\int_{-\infty}^{\infty} F\left(\mu_{p}\right) p d f(p) d p \\
\quad \approx \int_{-\infty}^{\infty}\left[F\left(\mu_{p}\right)+\left.\frac{\partial F}{\partial p}\right|_{p=\mu_{p}}\left(p-\mu_{p}\right)+\left.\frac{\partial^{2} F}{\partial p^{2}}\right|_{p=\mu_{p}}\left(p-\mu_{p}\right)^{2}\right] p d f(p) d p \\
\quad=F\left(\mu_{p}\right)+\left.\frac{1}{2} \frac{\partial^{2} F}{\partial p^{2}}\right|_{p=\mu_{p}} \sigma_{p}^{2}
\end{aligned}
$$

(e.g. Papoulis, 1991), where $p d f(p)$ is the probability density function of $p$ (in this case it is the normal distribution $\left.N\left(\mu_{p}, \sigma_{p}\right)\right)$, and $\left.\frac{\partial F}{\partial p}\right|_{p=\mu_{p}}$ and $\left.\frac{\partial^{2} F}{\partial p^{2}}\right|_{p=\mu_{p}}$ are first and second derivatives of $F$ with respect to the land surface parameter $p$. As the first moment is approximated in second order accuracy, the approach is referred to as Second-Order-FirstMoment propagation (SOFM).

The sensitivities are obtained analytically, as the SVATmodel is programmed in Mathematica, which allows algebraic computation of derivatives, even for complex equations as it is in case of the numerical solution of the surface energy equations and all equations hereafter.

The effective parameter $p_{\text {eff }}$ is based on the solution of

$F\left(\mu_{p}\right)+\left.\frac{1}{2} \frac{\partial^{2} F}{\partial p^{2}}\right|_{p=\mu_{p}} \sigma_{p}^{2} \stackrel{!}{=} F\left(p_{\mathrm{eff}}\right)$

which requires determining the root of

$F\left(\mu_{p}\right)+\left.\frac{1}{2} \frac{\partial^{2} F}{\partial p^{2}}\right|_{p=\mu_{p}} \sigma_{p}^{2}-F\left(p_{\text {eff }}\right)=0$ 

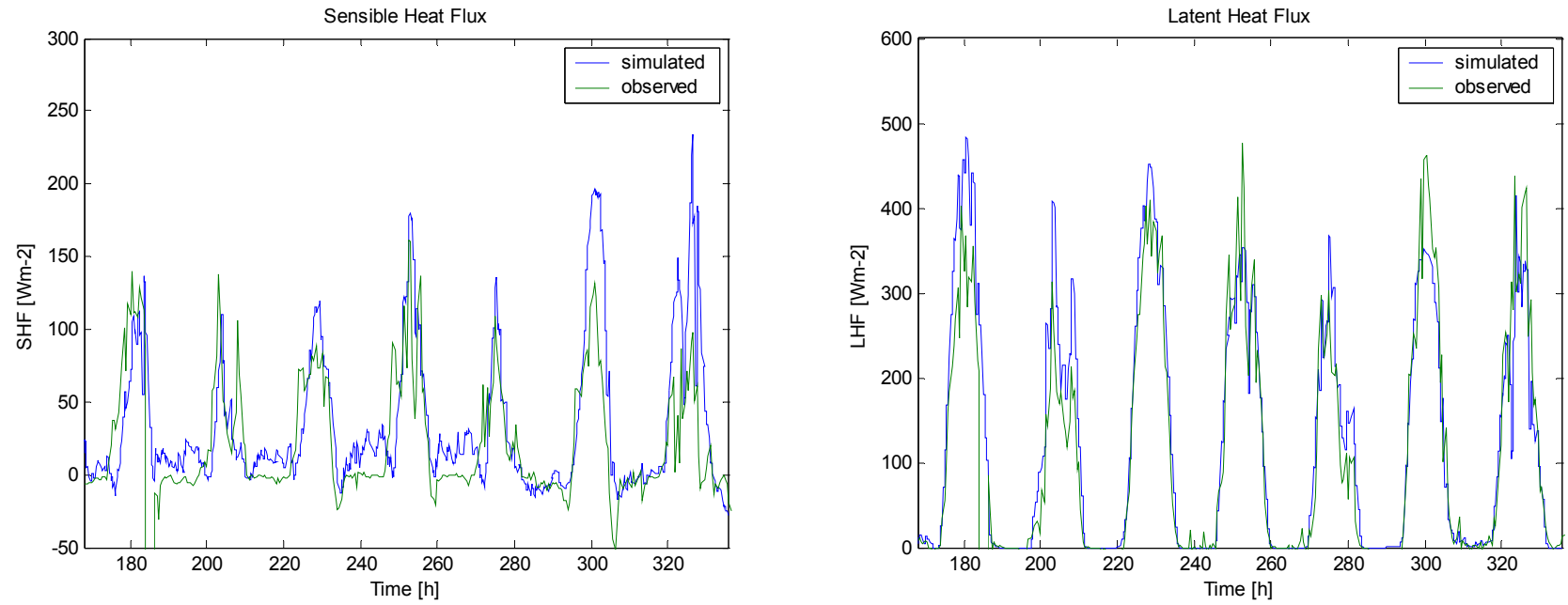

Fig. 1. Validation of SVAT-model: comparison between modelled and simulated sensible heat fluxes (a, left) and latent heat fluxes (b, right).

Subgrid scale

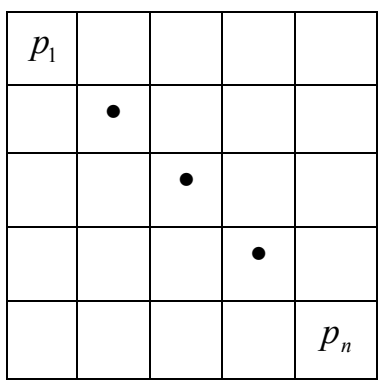

Land surface parameters $p_{i}(i=1, . ., n)$

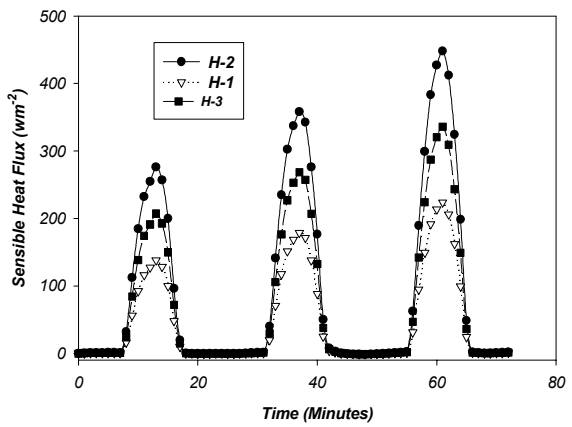

Subgrid scale fluxes: $\mathrm{F}_{\mathrm{i}}\left(p_{i}\right)$
Grid scale

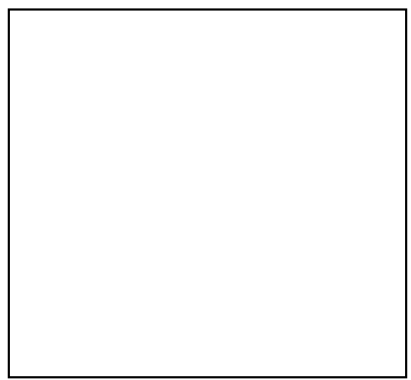

Effective parameter, $p_{\text {eff }}$

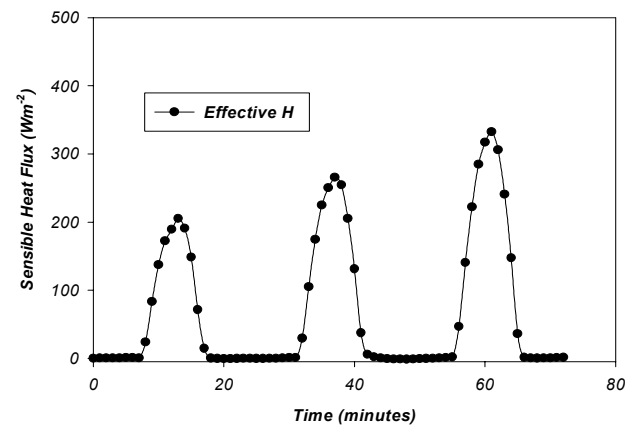

Grid scale fluxes: $\mathrm{F}\left(p_{\text {eff }}\right)$

Fig. 2. Schematic presentation of definition of effective parameter $p_{\text {eff }}$ and aggregation of heat fluxes (adapted from Intsiful, 2004).

This is easily achieved by standard packages within Mathematica.

For every given set of mean $\mu_{p}$ and standard deviation $\sigma_{p}$ at subgrid scale, the solution of the root finding problem (8) has to be solved. The effective parameter then finally is mapped as a function of $\mu_{p}$ and $\sigma_{p}$ :

$$
p_{\text {eff }}=f\left(\mu_{p}, \sigma_{p}\right)
$$




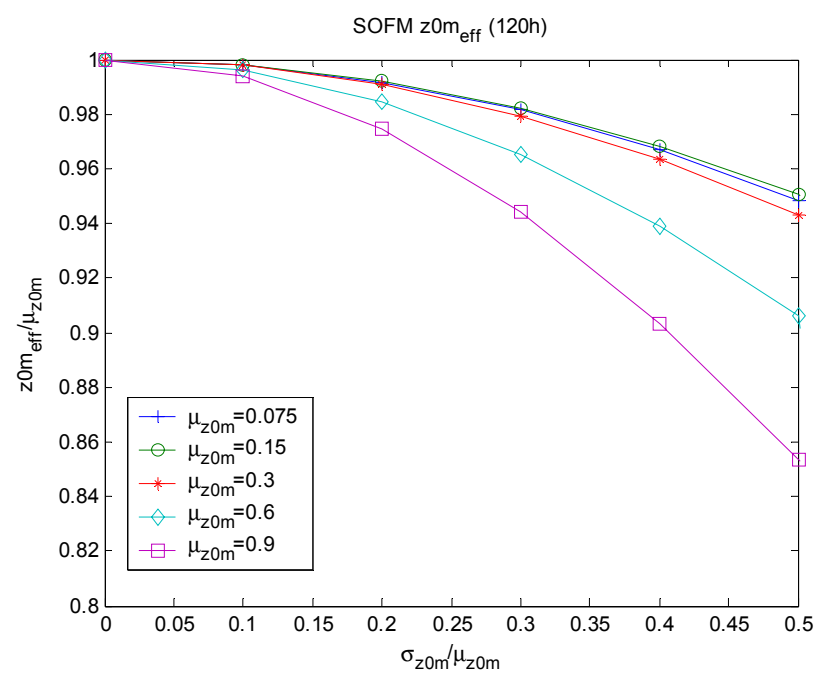

Fig. 3. Upscaling relations for roughness length $z_{0 \mathrm{~m}}$ (SOFM method).

\section{Results}

The proposed new approach for upscaling of land surface parameters is demonstrated for the parameters a) roughness length, b) wilting point soil moisture, and c) minimal stomata resistance. For the objective function to be met at grid scale, $\lambda E$ (latent heat flux) was chosen for $F$ in (4) till (8). The upscaling relations are visualised by plotting the normalised effective parameter (i.e. $p_{\text {eff }} / \mu_{p}$ ) against the coefficient of variation (i.e. $\sigma_{p} / \mu_{p}$ ) for different mean parameter values $\mu_{p}$. Figure 3 shows the derived upscaling relation for roughness length $z_{0 \mathrm{~m}}$. It is seen that with increasing subgrid scale heterogeneity (i.e. coefficient of variation) the effective roughness length is decreasing. Figure 4 shows the results of a corresponding Monte Carlo simulation. In fact the two approaches yield comparable results. Only in case of larger coefficients of variation, the Monte Carlo simulation differs.

Effective parameters must be independent of the driving meteorology. The central question is: what is the minimum duration (episode) such that the derived value for the effective parameter converges and becomes independent as time proceeds. Figure 5 shows the dependency of the derived effective roughness length in dependence of simulation time. After around $80 \mathrm{~h}$ the derived effective value converges. It is therefore concluded that $120 \mathrm{~h}$ simulation time is sufficient for deriving effective land surface parameters in this study. It must be noted, however, that the effective parameters may still depend on regional climatology and the season of the year. A further investigation in this direction, however, is out of the scope of this study.

Figure 6 shows the upscaling relations in case of wilting point soil moisture $\theta_{\text {wilt }}$. Contrary to the case of roughness length, effective values increase with increasing coefficient of variation.

Figure 7 shows the upscaling relations for the vegetation type dependent minimum stomatal resistance $R c_{\min }$. The

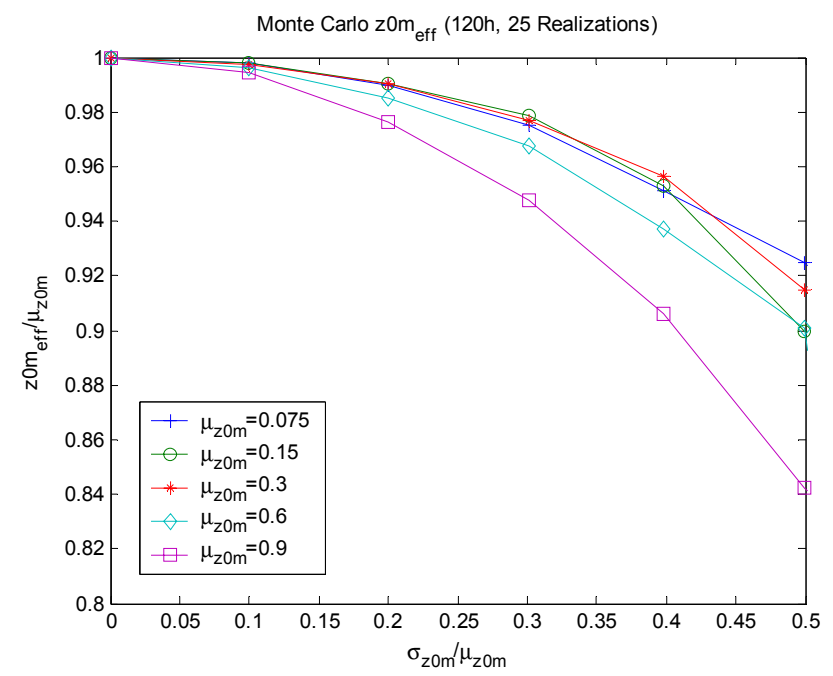

Fig. 4. Upscaling relations for roughness length $z_{0 \mathrm{~m}}$ (Monte Carlo method, 25 realizations)



Fig. 5. Dependency of derived effective parameter on simulation time in case of roughness length $z_{0 \mathrm{~m}}$ : convergence after $60-80 \mathrm{~h}$.

general shape of the scaling laws is similar to the case of roughness length.

\section{Summary and conclusion}

A new approach for the derivation of effective land surface parameters was presented. It was shown that the methodology yields results that are in excellent agreement with corresponding Monte Carlo results. The SOFM approach, however, is much less computational demanding than the Monte Carlo approach. This is also due to the fact that the programming environment (Mathematica) chosen for this study allows the computationally efficient algebraic calculation of derivatives. 


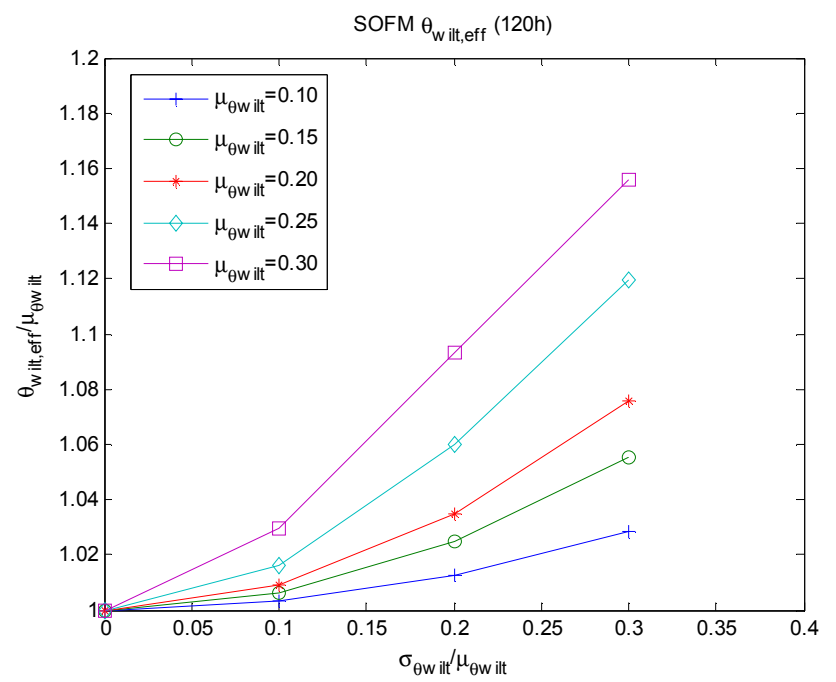

Fig. 6. Upscaling relations for wilting point soil moisture $\theta_{\text {wilt }}$ (SOFM method).

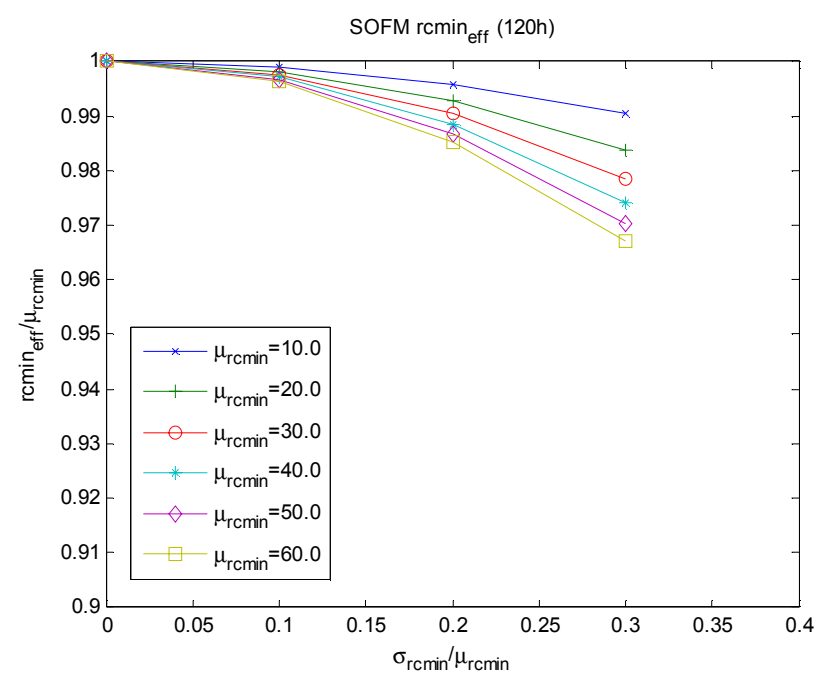

Fig. 7. Upscaling relations for minimum stomatal resistance $R c_{\min }$ (SOFM method).

Compared to other upscaling methods (e.g. Hu et al., 1999), the proposed methodology is independent of driving meteorology. It is concluded that the methodology proposed can also be advantageously applied in other areas, such as hydrogeology for example.
Acknowledgements. This work was performed within the GLOWAVolta project (http://www.glowa-volta.de) funded by the Germany Ministry of Education and Science (BMBF). The financial support is gratefully acknowledged.

Edited by: P. Krause, K. Bongartz, and W.-A. Flügel

Reviewed by: anonymous referees

\section{References}

Ek, M. and Mahrt, L.: OSU 1-D PBL Model User's Guide Version 1.0.4, Department of atmospheric Sciences, Oregon State University, 1991.

Chen, F. and Dudhia, J.: Coupling an Advanced LandSurface/Hydrology Model with the Penn State/NCAR MM5 Modeling System, Part I: Model Implementation and Sensitivity, Monthly Weather Review, 129, 569-585, 2001.

Grell, G., Dudhia, J., and Stauffer, D.: A Description of the FifthGeneration Penn State/NCAR Mesoscale Model (MM5), NCAR Technical Note, NCAR/TN-398+STR, pp. 117, 1994.

$\mathrm{Hu}$, Y., Islam, Y., and Jiang, L.: Approaches for aggregating heterogeneous surface parameters and fluxes for mesoscale and climate models, Boundary Layer Meteorology, 93, 313-336, 1999.

Intsiful, J.: Upscaling of Land Surface Parameters through Inverse SVAT modelling, PhD at Bonn University, published in Ecology and Development Series, No. 20, Cuvillier Verlag Göttingen, ISBN 3-86537-174-4, 2004.

Papoulis, A.: Probability, Random Variables and Stochastic Processes, McGraw Hill, 1991.

Wolfram, S.: Mathematica 5.1, Wolfram Research Inc., 2004. 